\title{
Saccharomyces Boulardii in Helicobacter Pylori Eradication in Children: A Randomized Trial From Iran
}

\author{
Kokab Namkin, ${ }^{1}$ Mahmood Zardast, ${ }^{2}$ and Fatemeh Basirinejad ${ }^{2, *}$ \\ ${ }^{1}$ Department of Pediatrics, Valieasr Hospital, Birjand University of Medical Sciences, Birjand, IR Iran \\ ${ }^{2}$ Valieasr Hospital, Birjand University of Medical Sciences, Birjand, IR Iran \\ ${ }^{*}$ Corresponding author: Fatemeh Basirinejad, Valieasr Hospital, Birjand University of Medical Sciences, Birjand, IR Iran. Tel: +98-5136057487, Fax: +98-5136093201, \\ E-mail: fatemehbasirinejad@gmail.com
}

Received 2015 August 6; Revised 2015 October 21; Accepted 2015 November 3.

\begin{abstract}
Background: Helicobacterpylori infects around $50 \%$ of the human population and is asymptomatic in $70 \%$ of the cases. H. pylori eradication in childhood will not only result in peptic symptoms relief, but will also prevent late-term complications such as cancer. Today, probiotics are being increasingly studied in the treatment of gastrointestinal infections as an alternative or complement to antibiotics.

Objectives: In this study we aimed to assess the effect of S. boulardii supplementation on H. pylori eradication among children in our region.

Patients and Methods: In this randomized double-blind placebo-controlled clinical trial 28 asymptomatic primary school children with a positive $H$. pylori stool antigen (HpSA) exam were randomly allocated into the study group, receiving Saccharomyces boulardii, and the control group receiving placebo capsules matched by shape and size, for one month. The children were followed up weekly and were reinvestigated four to eight weeks after accomplished treatment by HpSA testing. The significance level was set at $\mathrm{P}<0.05$.

Results: 24 children completed the study. The mean HpSA reduced from $0.40 \pm 0.32$ to $0.21 \pm 0.27$ in the study group, indicating a significant difference $(\mathrm{P}=0.005)$. However, such difference was not observed in the control group $(\mathrm{P}=0.89)$. Moreover, the HpSA titer showed a $0.019 \pm$ 0.19 decrease in the study group whereas the same value was $0.0048 \pm 0.12$ for the controls, again stating a significant difference $(\mathrm{P}=0.01)$. Conclusions: Saccharomyces boulardii has a positive effect on reducing the colonization of $H$. pylori in the human gastrointestinal system but is not capable of its eradication when used as single therapy.
\end{abstract}

Keywords: Probiotic, Helicobacter Pylori Stool Antigen, Saccharomyces Boulardii, Helicobacter Pylori

\section{Background}

Helicobacter pylori is a Gram negative, spiral-shaped pathogen infecting an estimated 50\% of the human population which is asymptomatic in about $70 \%$ of the cases (1-3). It has a much higher prevalence in developing countries $(1,2)$, most probably as a consequence of different hygiene and living conditions, and the uncontrolled use of antibiotics (4).

Childhood is the period in which most H. pylori infections tend to be acquired especially in developing nations $(5,6)$. H. pylori is considered to be the major cause of chronic gastritis and duodenal ulcer in childhood and an important cofactor in the development of gastric cancer or mucosa-associated lymphoid tissue (MALT) lymphoma $(7,8)$. Therefore, $H$. pylori eradication in childhood will not only result in peptic symptoms relief, but will also prevent late-term complications, such as cancer (9).

For $H$. pylori diagnosis either invasive (pathology, culture, RUT) or noninvasive techniques (urea breath test, serology, H. pylori stool antigen) may be used. As for developing countries which have a higher prevalence of this infection, lower-cost non-invasive techniques are preferred; among them is the H. pylori stool antigen (HPSA) testing.

In a study conducted in Iran the sensitivity and specificity of $H$. pylori stool antigen (HpSA) was estimated as $85 \%$ and 93\%, respectively (10). The same values were reported as $96.3 \%$ and $95.1 \%$ in another study from Mexico (11). Therefore, regarding the high sensitivity and specificity of HpSA, this test can be used as an alternative technique for $H$. pylori infection diagnosis in developing countries.

So far therapy failure has been reported in more than $30 \%$ of childhood cases, mainly due to low compliance and an increase in the prevalence of antibiotic-resistant bacteria $(2,8)$. Moreover, as most of the colonized children remain asymptomatic, antibiotic therapy is not ethically acceptable. High costs for low socioeconomic families (the most affected cases) and the relative inefficiency of the this type of treatment due to the tendency for rapid re-colonization are other limiting factors in developing countries $(12,13)$. Therefore the possibility to re-

Copyright (C) 2016, Growth \& Development Research Center. This is an open-access article distributed under the terms of the Creative Commons Attribution-NonCommercial 4.0 International License (http://creativecommons.org/licenses/by-nc/4.0/) which permits copy and redistribute the material just in noncommercial usages, provided the original work is properly cited. 
duce this phenomenon by the simple administration of a probiotic seems quite fascinating.

Probiotics are live organisms or produced substances which are orally administered and can have beneficial effects on the host (2). The main mechanisms by which probiotics specifically influence $H$. pylori are the production of antimicrobial substances; they strengthen the non-immunological barriers representing as first line of defense against pathogenic bacteria along with stabilization of the gut mucosal barrier $(3,14)$.

Clinical trials evaluating the effect of probiotics on $H$. pylori infection have indicated that probiotics alone are generally incapable of eradicating $H$. pylori, but may decrease its density in the stomach (15). Dinleyici et al. (16) reported that Saccharomyces boulardii improves compliance, decreases side effects, and moderately increases the eradication rate of $H$. pylori while Riaz et al. (17) showed positive effects of $S$. boulardii in acute childhood diarrhea. However, Hurduc et al. (18) using the S. boulardii strain, reported no effect on $H$. pylori eradication rate, yet with beneficial health effects in the infected cases.

Nevertheless, probiotics are being increasingly studied in the treatment of gastrointestinal infections as an alternative or complement to antibiotics, with the potential to decrease the use of antibiotics or reduce their side effects (19).

\section{Objectives}

Considering the rarity of a comprehensive survey on the potential role of probiotics for the treatment of childhood $H$. pylori infection in Iran; in this study, we aimed to assess the effect of $S$. boulardii supplementation on $H$. pylori eradication among children.

\section{Patients and Methods}

In this randomized double-blind placebo-controlled clinical trial performed from March-Sept 2014, 28 asymptomatic primary school children with a positive stool exam for $H$. pylori antigens were enrolled. The children were selected from the positive cases diagnosed in an extensive screening program on the prevalence of $H$. pylori infection among school children in Birjand, Iran by the same authors (20).

The children were first assessed for eligibility criteria. Individuals with severe gastrointestinal signs and symptoms requiring further treatment, having received antibiotic therapy during the past month, with a history of antacid usage (PPI or $\mathrm{H} 2$ blocker), diarrhea at the time of sampling, history of immunodeficiency disorders, and any positive history of food or drug allergy were excluded from the study. Fortunately, none of the 28 selected cases had any of the exclusion criteria. The study protocol was then fully explained to the children and their parents/ guardians and an informed consent was signed by the parents of each child prior to study entrance. The study protocol was fully approved by the Ethics Committee of Birjand University of Medical Sciences.
The participants (all aged 9 - 12 years) were randomly allocated into two groups. The study group received $S$. boulardii, a well-known probiotic which is marketed under the brand of Yomogi. Each capsule contained 250 milligram of lyophilized S. boulardii. The control group received placebo capsules with the same shape, size and color but containing lactose and wheat starch powder. Both capsules were in packs of 30 and were prescribed daily for one month. Both the research team and the patients were blind to the contents of the prescribed packs during the study. The patients were followed up weekly by a telephone call made by a research fellow who was also blind to patients' allocation. The number of capsules consumed in the past week, possible side effects, time and route of consumption (swallowing or opening the capsule and mixing its contents with yogurt or water) and simultaneous use of other drugs were questioned and recorded. In case of any adverse events the parents were asked to visit the Gastroenterology clinic for precise management and follow up. In addition, we gave a phone number to all parents to contact us if needed.

All patients were reinvestigated four to eight weeks after accomplished treatment by HpSA testing. Successful treatment was defined as a negative HpSA test result. Stool sampling test was performed with the same kit used in the previous screening (H pylori Antigen EIA Test Kit, ACON company). The questionnaire was also once again filled for each participant. According to similar studies, consumption of $\geq 80 \%$ of the prescribed drug was considered as good enough. The HpSA level was measured quantitatively in microgram per milliliter unit. The basis of this test is the Sandwich method which is based on the formation of antigen-antibody complexes and detection of bound material by spectrophotometry. The kit used was an approved kit for quantitative detection of $H$. pylori antigen in human stools with the ELISA method using monoclonal antibodies. The kit sensitivity was $98.6 \%$ and its specificity was $95.4 \%$.

The data were analyzed with IBM SPSS statistics for Windows version 21.0. Chi-square test and Mann-Whitney test were applied wherever appropriate. The significance level was set at $\mathrm{P}<0.05$.

\section{Results}

In total 28 participants were studied, 14 in each group. 4 cases were excluded during data analysis due to certain errors and therefore the data of 24 cases were fully analyzed (Figure 1). $16.7 \%$ and $25 \%$ of the cases in the study and control group were male and the rest were female, respectively.

In each group five participants did not consume all 30 prescribed capsules due to either disremembering or inaccessibility. Considering different possible side effects including fever, diarrhea, nausea and vomiting, abdominal pain, etc, only one (8.3\%) case of loss of appetite was recorded in the study group. 
As demonstrated in Table 1, the mean HpSA reduced from $0.40 \pm 0.32$ to $0.21 \pm 0.27$ in the study group, indicating a significant difference $(\mathrm{P}=0.005)$. However no such difference was observed in the control group $(\mathrm{P}=0.89)$. Moreover, the HpSA titer showed a $0.019 \pm 0.19$ decrease in the study group whereas the same value was $0.0048 \pm$
0.12 for the controls, again stating a significant difference $(\mathrm{P}=0.01$ ) (Figure 2).

The mean number of capsules consumed in the study and control group was $28.75 \pm 1.96$ and $28.58 \pm 2.2$, respectively. Mann-Whitney test showed no significant difference between the two groups in this respect $(\mathrm{P}=0.932)($ Table 1$)$.

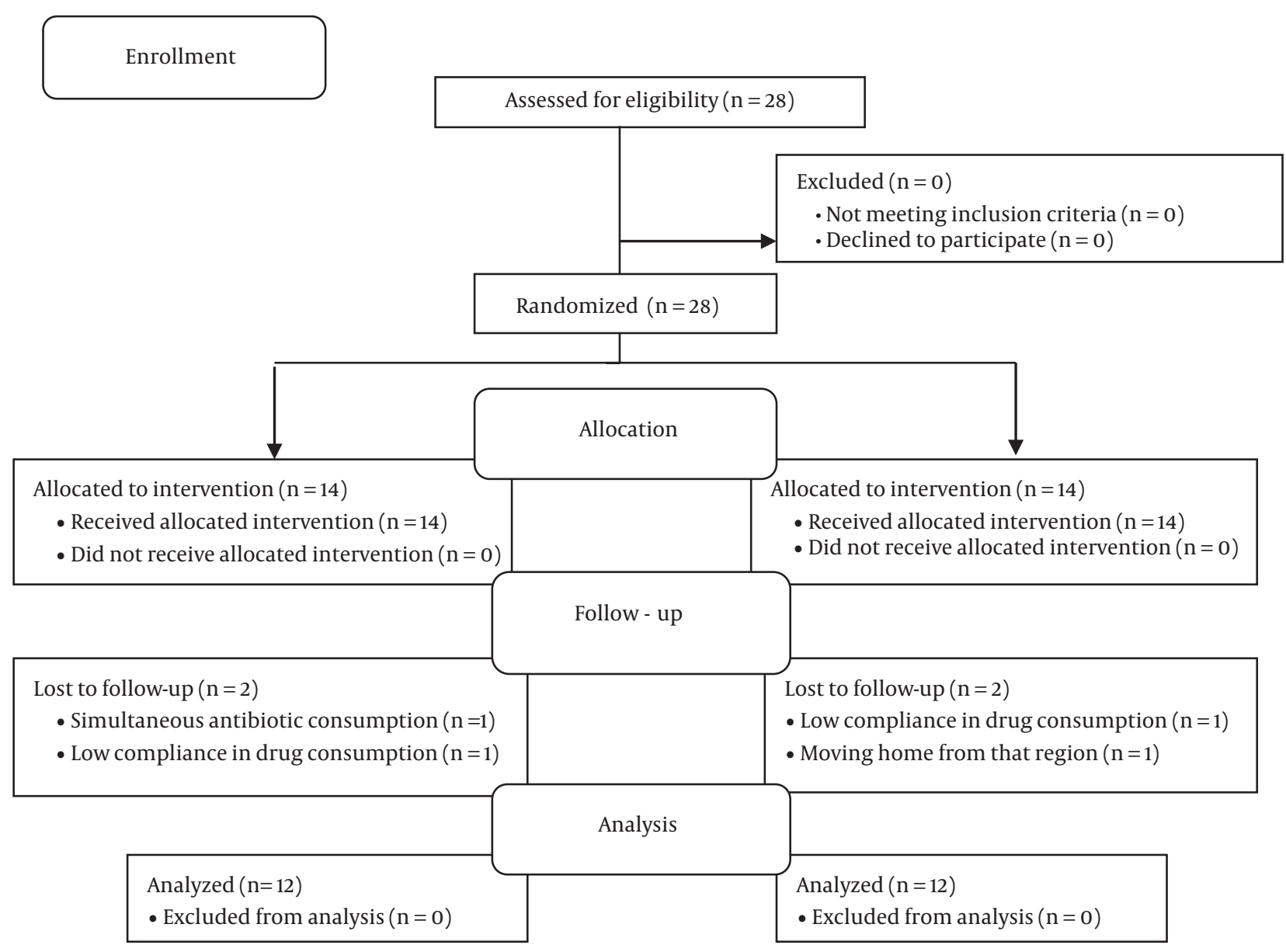

Figure 1. Flow Chart of the Studied Cases

Table 1. Comparing the Mean HpSA Titer Before and After Treatment in the Two Studied Groups

\begin{tabular}{lcccc}
\hline HpSA Titer & $\begin{array}{c}\text { Before } \\
\text { Intervention }\end{array}$ & $\begin{array}{c}\text { After } \\
\text { Intervention }^{\mathrm{a}, \mathrm{b}}\end{array}$ & $\begin{array}{c}\text { Wilcoxon Signed-Rank Test } \\
\text { Result }\end{array}$ \\
\hline Study group & $0.4 \pm 0.32$ & $0.21 \pm 0.27$ & $0.005^{\mathrm{C}}$ & $-\begin{array}{c}\text { Antibody Titer } \\
\text { Changes }\end{array}$ \\
$\begin{array}{l}\text { Control group } \\
\text { P-value }^{\mathrm{C}}\end{array}$ & $0.24 \pm 0.2$ & $0.24 \pm 0.27$ & 0.89 & $-0.19 \pm 0.19$ \\
\hline
\end{tabular}

Abbreviation : HpSA, Helicobacter pylori stool antigen.

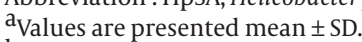

$\mathrm{b}_{\mathrm{N}=12 \text {. }}$

${ }^{\mathrm{C}}$ Mann-Whitney test result. 


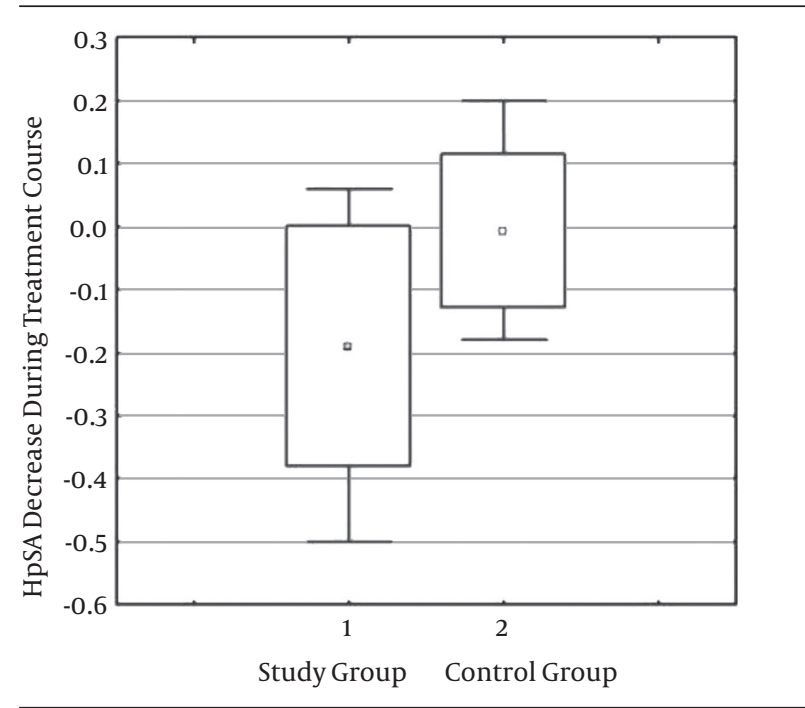

Figure 2. Comparing the Decrease Rate of H. pylori Stool Antigen Between the Study and Control Groups

\section{Discussion}

To date, numerous treatment regimens have been attempted to eradicate $H$. pylori; however, the success rates are far from ideal, ranging from $50 \%$ in developing countries (21) to $75 \%$ in the developed world (22). The most widely accepted first line treatment regimen includes the combination of a proton pump inhibitor and two antibiotics (amoxicillin and clarithromycin).

As some $H$. pylori strains are antibiotic-resistant, resulting in therapeutic failure, the development of alternative non-antibiotic approaches to treat $H$. pylori and other pathogenic bacteria seems essential.

Adjuvant therapy with probiotics has recently been widely used in $H$. pylori eradication. Various studies have administered different strains and doses of probiotics, and yielded conflicting results. In a brief review of $12 \mathrm{hu}$ man studies investigating the efficacy of combinations of antibiotics and probiotics, and 16 other studies using probiotics alone as an alternative to antibiotics for the treatment of $H$. pylori infection, improvement of $H$. pylori gastritis and decrease in $H$. pylori colonization after administration of probiotics was reported. However, no study achieved complete eradication of $H$. pylori infection by probiotic treatment alone (18).

Similar studies in children are limited. In the present study we aimed at investigating the effect of Saccharomyces boulardii on the eradication of $H$.pylori in school-aged children in Birjand, Iran. No significant difference was observed between the two groups regarding the eradication rate $(\mathrm{P}=0.16)$; however, the decrease in HpSA concentration was significantly higher in the probiotic-treated group $(\mathrm{P}=0.005$ vs $\mathrm{P}=0.89$ ).

In similar studies by Khodadad et al. (1) and Lesbros et al. (23), the rate of $H$. pylori eradication was significantly higher in the probiotic plus antibiotic-therapy group, in disagreement to our findings.

In a newly published study by Bin et al. (24) on H. pylori positive children, $S$. boulardii was added to triple therapy in the study group while the control group received triple therapy alone for two weeks. Urea breath test showed successful $H$. pylori eradication in $71.4 \%$ of the patients in the treatment group and in $61.9 \%$ of the control group, being statistically insignificant. However, S. boulardii revealed a beneficial effect on the prevention and treatment of diarrhea during $H$. pylori eradication and despite the slight increase in the eradication rate, S. boulardii improved compliance to eradication treatment.

In another study on 100 pediatric cases in 2014, standard H. pylori eradication treatment was added to Lactobacillus acidophilus and Bifidobacterium lactis, leading to a significantly higher eradication rate compared to the controls ( $83.7 \%$ vs $64.4 \%$ ) with no significant difference in terms of side effects (25).

However, in a recent study by Akcam et al. (26) from Turkey on 61 symptomatic children (aged 7 - 18 years) with H. pylori infection, the addition of probiotics to standard triple therapy influenced neither the eradication rate nor the adverse effects. In a recent study conducted in Tehran, Iran, 66 H. pylori positive children were treated with a triple drug treatment protocol (omeprazole + amoxicillin + furazolidon) and randomly allocated to receive either probiotic or placebo. HpSA was assessed after 4 - 8 weeks of treatment completion. Its eradication rate was significantly higher in the probiotic group $(\mathrm{P}=0.04)$ with a lower rate of side effects (1).

Taken together, all above mentioned studies investigated the effect of probiotics along with $H$. pylori triple therapy. Although the antibiotic regimen had been similar for both the study and control groups, yet the synergistic effect of probiotics on antibiotics leading to a desired gastrointestinal microenvironment for the absorption and function of antibiotics may have led to such higher eradication rates. To date, only a small number of studies have been conducted on the single effect of probiotics on H.pylori, similar to the current study, mostly reporting partial efficacy.

Recently, Szajewska et al. (27) presented an interesting meta-analysis involving a total of 1307 participants (among them only 90 children). S. boulardii given along with triple therapy significantly increased $H$. pylori eradication rate and reduced the risk of overall $H$. pylori therapy related adverse effects, particularly diarrhea.

Regarding limitations, the present study had a small sample size and $H$. pylori culture was not performed. In addition, only the presence or absence of side effects was considered without scoring symptom severity.

\subsection{Conclusion}

Saccharomyces boulardii has a positive effect in reducing the colonization of $H$. pylori in the human gastroin- 
Namkin Ket al.

testinal system but is not capable of $H$. pylori eradication when used as single therapy. Complementary studies with various durations and yeast dosages are highly recommended. Whether other probiotic strains have beneficial effects also needs to be substantiated in further randomized trials.

\section{Acknowledgments}

The present study was derived from a student thesis for obtaining a specialty degree in Pediatrics (IRCT code: 2014052117791N1). The authors would like to thank the Research Council of Birjand University of Medical Sciences for supporting the study financially.

\section{References}

1. Khodadad A, Farahmand F, Najafi M, Shoaran M. Probiotics for the treatment of pediatric Helicobacter pylori infection: a randomized double blind clinical trial. Iran J Pediatr. 2013;23(1):7984. [PubMed: 23446685]

2. Mehling H, Busjahn A. Non-viable Lactobacillus reuteri DSMZ 17648 (Pylopass) as a new approach to Helicobacter pylori control in humans. Nutrients. 2013;5(8):3062-73. doi: 10.3390| nu5083062. [PubMed: 23917169]

3. Vitor JM, Vale FF. Alternative therapies for Helicobacter pylori: probiotics and phytomedicine. FEMS Immunol Med Microbiol. 2011;63(2):153-64. doi: 10.1111/j.1574-695X.2011.00865.x. [PubMed: 22077218]

4. Ruggiero P. Use of probiotics in the fight against Helicobacter pylori. World J Gastrointest Pathophysiol. 2014;5(4):384-91. doi 10.4291/wjgp.v5.i4.384. [PubMed: 25400981]

5. Horvitz G, Gold BD. Gastroduodenal diseases of childhood. Curr Opin Gastroenterol. 2006;22(6):632-40. doi: 10.1097/01. mog.0000245534.58389.f3. [PubMed:17053441]

6. Boonyaritichaikij S, Kuwabara K, Nagano J, Kobayashi K, Koga Y Long-term administration of probiotics to asymptomatic preschool children for either the eradication or the prevention of Helicobacter pylori infection. Helicobacter. 2009;14(3):202-7. doi: 10.1111/j.1523-5378.2009.00675.x. [PubMed:19702850]

7. Uemura N, Okamoto S, Yamamoto S, Matsumura N, Yamaguch $\mathrm{S}$, Yamakido M, et al. Helicobacter pylori infection and the development of gastric cancer. N Engl J Med. 2001;345(11):784-9. doi: 10.1056/NEJMoa001999. [PubMed:11556297]

8. Lionetti E, Indrio F, Pavone L, Borrelli G, Cavallo L, Francavilla R. Role of probiotics in pediatric patients with Helicobacter pylori infection: a comprehensive review of the literature. Helicobacter. 2010;15(2):79-87. doi: 10.1111/j.1523-5378.2009.00743.x. [PubMed: 20402810]

9. Akcam M, Elmas O, Yilmaz A, Caglar S, Artan R, Gelen T, et al. Myeloperoxidase, xanthine oxidase and superoxide dismutase in the gastric mucosa of Helicobacter pylori positive and negative pediatric patients. Mol Cell Biochem. 2006;290(1-2):125-30. doi: 10.1007/s11010-006-9176-9. [PubMed:16758302]

10. Iranikhah A, Ghadir MR, Sarkeshikian S, Saneian H, Heiari A, Mahvari M. Stool antigen tests for the detection of Helicobacter pylori in children. Iran J Pediatr. 2013;23(2):138-42. [PubMed: 23724172]

11. Sethi A, Chaudhuri M, Kelly L, Hopman W. Prevalence of Helicobacter pylori in a First Nations population in northwestern Ontario. Can Fam Physician. 2013;59(4):e182-7. [PubMed: 23585621]
12. Magista AM, Ierardi E, Castellaneta S, Miniello VL, Lionetti E, Francavilla A, et al. Helicobacter pylori status and symptom assessment two years after eradication in pediatric patients from a high prevalence area. J Pediatr Gastroenterol Nutr. 2005;40(3):3128. [PubMed: 15735485$]$

13. Elitsur Y, Yahav J. Helicobacter pylori infection in pediatrics. Helicobacter. 2005;10 Suppl 1:47-53. doi: 10.1111/j.15235378.2005.00332.x. [PubMed:16178971]

14. Garcia CA, Henriquez AP, Retamal RC, Pineda CS, Delgado Sen C, Gonzalez CC. [Probiotic properties of Lactobacillus spp isolated from gastric biopsies of Helicobacter pylori infected and noninfected individuals]. Rev Med Chil. 2009;137(3):369-76. [PubMed: 19621178]

15. Gotteland M, Brunser O, Cruchet S. Systematic review: are probiotics useful in controlling gastric colonization by Helicobacter pylori? Aliment Pharmacol Ther. 2006;23(8):1077-86. doi: 10.1111/j.1365-2036.2006.02868.x. [PubMed:16611267]

16. Dinleyici EC, Kara A, Ozen M, Vandenplas Y. Saccharomyces boulardii CNCM I-745 in different clinical conditions. Expert Opin Biol Ther. 2014;14(11):1593-609. doi: 10.1517/14712598.2014.937419. [PubMed: 24995675]

17. Riaz M, Alam S, Malik A, Ali SM. Efficacy and safety of Saccharomyces boulardii in acute childhood diarrhea: a double blind randomised controlled trial. Indian J Pediatr. 2012;79(4):478-82. doi: 10.1007/s12098-011-0573-Z. [PubMed: 21997865]

18. Hurduc V, Plesca D, Dragomir D, Sajin M, Vandenplas Y. A randomized, open trial evaluating the effect of Saccharomyces boulardii on the eradication rate of Helicobacter pylori infection in children. Acta Paediatr. 2009;98(1):127-31. doi:10.1111/j.16512227.2008.00977.x. [PubMed: 18681892]

19. Patel A, Shah N, Prajapati JB. Clinical application of probiotics in the treatment of Helicobacter pylori infection--a brief review. J Microbiol Immunol Infect. 2014;47(5):429-37. doi: 10.1016/j. jmii.2013.03.010. [PubMed: 23757373]

20. Namakin K. Prevalence of Helicobacter Pylori Infection in Asymptomatic Children in Birjand, Eastern Iran. Int J Pediatr. 2014;2(4.2):55-63.

21. Tepes B, O'Connor A, Gisbert JP, O'Morain C. Treatment of Helicobacter pylori infection 2012. Helicobacter. 2012;17 Suppl 1:36-42. doi:10.1111/j.1523-5378.2012.00981.x. [PubMed: 22958154]

22. Federico A, Gravina AG, Miranda A, Loguercio C, Romano M. Eradication of Helicobacter pylori infection: which regimen first? World J Gastroenterol. 2014;20(3):665-72. doi: 10.3748/wjg.v20. i3.665. [PubMed: 24574740]

23. Lesbros-Pantoflickova D, Corthésy-Theulaz I, Blum AL. Helicobacter pylori and probiotics. J Nutr. 137:812-8.

24. Bin Z, Ya-Zheng X, Zhao-Hui D, Bo C, Li-Rong J, Vandenplas Y. The Efficacy of Saccharomyces boulardii CNCM I-745 in Addition to Standard Helicobacter pylori Eradication Treatment in Children. Pediatr Gastroenterol Hepatol Nutr. 2015;18(1):17-22. doi: 10.5223 pghn.2015.18.1.17. [PubMed:25866729]

25. Wang YH, Huang Y. Effect of Lactobacillus acidophilus and Bifidobacterium bifidum supplementation to standard triple therapy on Helicobacter pylori eradication and dynamic changes in intestinal flora. World J Microbiol Biotechnol. 2014;30(3):847-53. doi: 10.1007/s11274-013-1490-2. [PubMed: 24233772]

26. Akcam M, Koca T, Salman H, Karahan N. The effects of probiotics on treatment of Helicobacter pylori eradication in children. Saudi Med J. 2015;36(3):286-90. doi: 10.15537/smj.2015.3.10124. [PubMed: 25737169]

27. Szajewska H, Horvath A, Kolodziej M. Systematic review with meta-analysis: Saccharomyces boulardii supplementation and eradication of Helicobacter pylori infection. Aliment Pharmacol Ther.2015;41(12):1237-45. doi:10.1111/apt.13214. [PubMed:25898944] 